\title{
Reliability assessment of shallow foundations on undrained soils considering soil spatial variability
}

\author{
J.T. Simões ${ }^{\mathrm{a}}$, Luis C. Neves ${ }^{\mathrm{b}}$, Armando N. Antão ${ }^{\mathrm{a}, *}$, Nuno M. C. Guerra ${ }^{\mathrm{a}}$ \\ ${ }^{a}$ UNIC, Department of Civil Engineering, Faculdade de Ciências e Tecnologia, Universidade Nova de \\ Lisboa, Portugal \\ ${ }^{b}$ Resilience Engineering Research Group, Faculty of Engineering, University of Nottingham, UK
}

\begin{abstract}
Structural design using partial safety factors aims at achieving an homogeneous safety level in geotechnical design without the use of more complex reliability analysis. In this work, the different Design Approaches proposed by Eurocode 7 for shallow foundations resting on the surface of undrained soils are compared in terms of the resulting reliability indices. The influence of both centered and eccentric loads, as well as homogeneous and heterogeneous isotropic and anisotropic distributions for the variability of soil properties were investigated in the reliability analysis.

A finite element implementation of the upper bound theorem of limit analysis is combined with Latin Hypercube sampling to compute the probabilistic response of shallow foundations. Considering realistic probabilistic distributions for both permanent and live loads, First Order Reliability Method is used to calculate the reliability index of such structures designed according to the different Design Approaches present in Eurocode 7.

The results obtained show that the Eurocode 7 leads to satisfactory reliability indices, but that significant differences between Design Approaches exist.
\end{abstract}

Keywords: Reliability assessment, Eurocode 7, anisotropic soil spatial variability, upper bound theorem, bearing capacity, uncertainty of load eccentricity

\section{1. Introduction}

The partial safety factors method is a semi-probabilistic safety verification method 3 aiming at attaining uniform and consistent safety levels, without the complexity of ex-

4 plicit reliability analysis. That approach is employed in Eurocode 7 [11] for geotechnical

5 limit states through the use of three different Design Approaches. These approaches

6 should result in uniform safety levels, consistent with the target reliability indices pre-

7 scribed in Eurocode 0 [10]. However, the different Design Approaches proposed in Eu-

8 rocode 7 [11] can, potentially, result in structures with very different levels of safety, and

\footnotetext{
${ }^{*}$ Corresponding author

Email addresses: jtbsimoes@gmail.com (J.T. Simões), luis.neves@nottingham.ac.uk (Luis C. Neves), amna@fct.unl.pt (Armando N. Antão), nguerra@fct.unl.pt (Nuno M. C. Guerra)
} 
9 a reliability analysis can assess the consistency of the methods proposed in codes [32, 10 e.g.].

In the partial safety factors method, the large uncertainties present in geotechnical 12 design $[38,39,53$, e.g.] are considered through the use of nominal values of properties 13 and safety factors applied to loads, soil properties and resistances [11].

The present paper aims at evaluating the reliability of the bearing capacity of shallow foundations under undrained conditions. For such analysis, it is paramount to consider Kulhawy [38], uncertainties in soil properties result from (i) inherent variability, (ii) errors in the performed measurements and (iii) errors from transformation of experimeninhere ra also be considered, in particular in what regards the eccentricity of applied loads. In fact, due to uncertainty in the amplitude of different loads, random deviations in the point of application of the resulting load may arise, influencing the failure mode of the foundation and its safety.

The collapse load of foundations under these conditions can be computed using different numerical approaches. In this paper, bearing capacity is determined using a finite element implementation of the limit analysis upper bound theorem, the Sublim3D code, described in [45]. The ability of the mentioned implementation to compute upper bound solutions of various problems has been previously demonstrated $[44,43,4]$. This code uses a parallel algorithm which significantly reduces the computational time. Calculations were performed in a cluster with 104 cores.

The use of advanced numerical methods for computing the collapse load of foundations limits the use of gradient-based reliability methods (e.g.,, FORM - First Order Reliability Method - and SORM - Second Order Reliability Method), making simulation the most effective tool for reliability assessment. To assess the methodology proposed in Eurocode 7 [11], the reliability index of a set of shallow foundations designed according to its three Design Approaches $(D A)$ is evaluated. The foundations under analysis are continuous footings on soil responding in undrained conditions. For these, two-dimensional models are usually employed and, due to their lower computational cost, will also be used here. The spatial variability of the soil properties, and therefore its heterogeneity, can be considered using random fields [38, e.g.]. Random fields can be generated using a wide range of approaches $[15,20,16]$. To reduce the number of samples required for achieving acceptable results, Latin Hypercube sampling was employed in this work [34, 21, 36]. This method was also used by Cho and Park [13] to study the same geotechnical problem.

Based on the available information on the probabilistic properties of soil $[33,51,5,31$, $24,47,12,40,42,38,39,14,6$, e.g.], a parametric study varying these properties is presented.

The influence of the soil spatial variability on various geotechnical problems has already been investigated [19]. With respect specifically to shallow foundations, serviciability limit states have been considered for different cases: plane strain single footing $[37,19,1]$, plane strain double footings [17, 2] and 3D single and double footings [18, 19]; these contributions used vertical loading, but inclined loads were also considered [3]. Moreover, the ultimate limit state problem has been deeply studied in the last years. The ultimate limit state of shallow foundations resting on soils responding in undrained 
56 conditions have already been greatly investigated $[22,23,41,13,28,9,27]$ consider-

57 ing different probabilistic distributions for the undrained shear strength, adopting both

58 isotropic and anisotropic structures for the spatial correlations and using different meth-

59 ods to generate the random fields. Most of the works investigated only the case of a

6o vertical centered load [22, 23, 41, 13, 28, 27] and only a recent work [9] considered the

61 effect of combination of horizontal and vertical loads, together with moments. In gen-

62 eral, these works combined the use of conventional non-linear finite elements analyses or

63 numerical limit analyses with random field theory and Monte Carlo simulations.

64 The previously mentioned works have clearly shown that average bearing capacity

65 considering the soil spatial variability is smaller than the one calculating admitting an

66 homogeneous soil. However, the impact of spatial variability of soil on the safety of foun-

67 dations considering the different Design Approaches proposed in Eurocode 7 in unknown.

${ }_{68}$ Do the Design Approaches of Eurocode 7 meet the desired level of safety for a wide range

69 of foundations when the spatial variability is considered?

In the present paper, reliability analysis is used to assess the level of safety of shal71 low foundations under undrained conditions of the Design Approaches of Eurocode 7 72 considering soil heterogeneity and the uncertainty of the eccentricity of the load. The 73 soil heterogeneity is defined by the statistical properties of the undrained shear strength, ${ }_{74} c_{u}$ : mean, coefficient of variation and anisotropic correlation length. The uncertainty of 75 the load is considered through a statistical distribution of its eccentricity. The obtained 76 results are fitted to a range of probabilistic distributions for each set of parameters char77 acterizing the soil. These are then used to compute the reliability index using the First 78 Order Reliability Method (FORM) [48, 29] for foundations designed following Eurocode 79 [11].

\section{so 2. Problem definition}

The problem studied is a strip shallow foundation with width $B$ on the surface of 82 undrained soil, submitted to two load cases (centered and eccentric load), as presented sз in Figure 1. The eccentricity, $e_{B}$, of the vertical load is assumed to be described by a ${ }_{84}$ normal distribution, being for each load case defined as:

$85 \quad$ Load case 1: $e_{B} \sim \mathrm{N}(0 ; 3 B / 100)$

$86 \quad$ Load case $2: e_{B} \sim \mathrm{N}(B / 6 ; 3 B / 100)$

87 The mean eccentricity considered in Load case 2 corresponds to the maximum value

s8 for which, in the homogeneous case, the base of the footing remains under compression.

89 Therefore, the conclusions of the study are limited to these cases.

The bearing capacity of the foundation considering a two-dimensional plane strain problem and a homogeneous soil can be determined by:

$$
R=N_{c} \cdot c_{u} \cdot B^{\prime}
$$

where $N_{c}$ is a bearing capacity factor, equal to $2+\pi, c_{u}$ is the undrained shear strength and $B^{\prime}$ is the effective width, defined as:

$$
B^{\prime}=B-2 \cdot e_{B}
$$

9o For heterogeneous soils, no closed form expression for the bearing capacity can be defined, 91 and numerical methods must be used. As shown in Equation 1, the undrained shear 


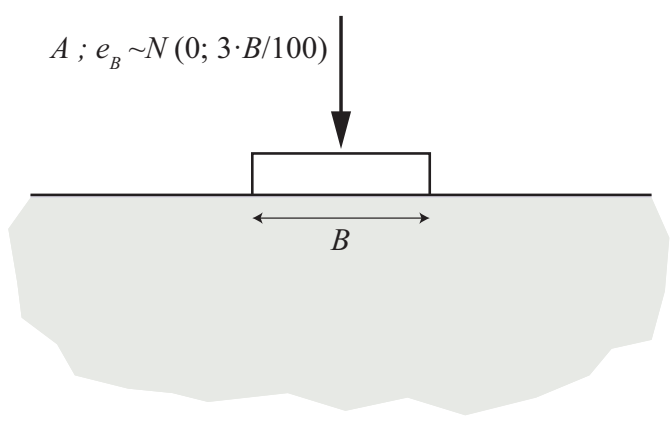

(a)

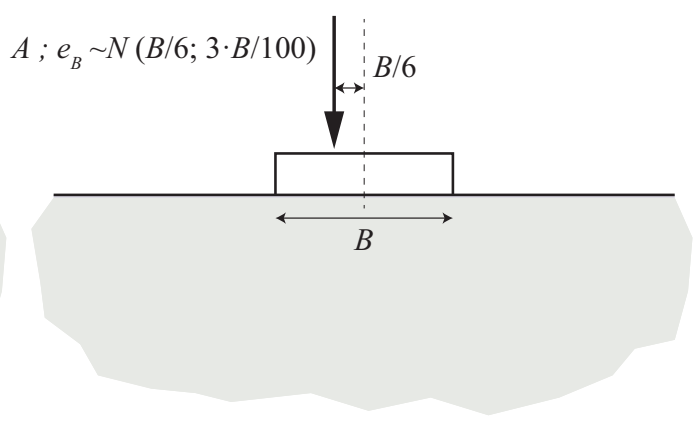

(b)

Figure 1: Scheme of the problem under study for centered (a) and eccentric load (b).

\section{Eurocode design}

The methodology proposed in Eurocode 7 [11] is based on the application of partial safety factors increasing the effects of actions and decreasing the material strengths or the resistances. The structural safety for a given limit state is satisfied when the design value of the resistance is higher than the corresponding effect of the design actions, $R_{d} \geq A_{d}$. For the present case, considering a homogeneous soil, the design value of resistance is given by:

$$
R_{d}=(2+\pi) \cdot \frac{c_{u, k}}{\gamma_{c_{u}}} \cdot B^{\prime} \cdot \frac{1}{\gamma_{R, v}}
$$

and the design value of the actions is defined as follows:

$$
A_{d}=\gamma_{G} \cdot G_{k}+\gamma_{Q} \cdot Q_{k}
$$

where $\gamma_{R, v}$ and $\gamma_{c_{u}}$ are the partial safety factors associated with the resistance and the undrained shear strength, respectively; $c_{u, k}$ is the characteristic value of the undrained shear strength; $G_{k}$ and $Q_{k}$ are the nominal values of permanent and live load, respectively, and $\gamma_{G}$ and $\gamma_{Q}$ are the partial safety factors associated with the permanent and live load, respectively.

Following Eurocode 7 [11], a set of limit states must be checked for shallow foundations. For the limit state GEO (failure or excessive deformation of the ground), under analysis in this work, Eurocode 7 [11] provides three alternative Design Approaches. These approaches combine different values for the partial safety factors aiming at a consistent safety level. For the structure under analysis, continuous footing on soil responding in undrained conditions, the Design Approaches are:

Design Approach 1 (DA1): 
Table 1: Partial factors for actions defined in Eurocode 7 [11] considering the GEO and STR ultimate states.

\begin{tabular}{cccc}
\hline Coefficient & Type & $A 1$ & A2 \\
\hline \multirow{2}{*}{$\gamma_{G}$} & Unfavorable & 1.35 & 1.00 \\
& Favorable & 1.00 & 1.00 \\
\hline \multirow{2}{*}{$\gamma_{Q}$} & Unfavorable & 1.50 & 1.30 \\
& Favorable & 0.00 & 0.00 \\
\hline
\end{tabular}

Table 2: Partial factors for vertical resistance of shallow foundations defined in Eurocode 7 [11] considering the GEO and STR ultimate states.

\begin{tabular}{cccccc}
\hline Structure & Resistance & Coefficient & $R 1$ & $R 2$ & $R 3$ \\
\hline Shallow Foundation & Bearing capacity & $\gamma_{R, v}$ & 1.00 & 1.40 & 1.00 \\
\hline
\end{tabular}

Table 3: Partial factors for undrained shear strength defined in Eurocode 7 [11] considering the GEO and STR ultimate states.

\begin{tabular}{ccc}
\hline Coefficient & $M 1$ & M2 \\
\hline$\gamma_{c_{u}}$ & 1.00 & 1.40 \\
\hline
\end{tabular}

Combination 1 (DA1.1): $A 1+M 1+R 1$

- Combination 2 (DA1.2): A2+M2+R1

Design Approach 2 (DA2): $A 1+M 1+R 2$

Design Approach 3 (DA3): (A1 or A2) $+M 2+R 3$

where "+" means "combined with" and A1, A2, M1, M2, R1, R2 and R3 are different sets of partial factors for actions $(A)$, material properties $(M)$ and resistance $(R)$. The values corresponding to these sets of partial factors are presented in Tables 1 to 3 .

and STR ultimate states.

The equations and partial factors presented show that $D A 3$, considering action $A 2$, is equivalent to $D A 1.2$, as for $R 1$ and $R 3$ the coefficients are both equal to 1.00. Moreover, as the foundation in the problem investigated is assumed to be subjected to vertical loads and to be located on the surface of a soil responding in undrained conditions, $D A 3$, considering action $A 1$, is equivalent to $D A 2$, since resistance is proportional to the undrained shear strength and, applying the safety factor with the same value (1.40) to the material properties or to the resistance is equivalent.

It should still be noted that in the case of the first Design Approach DA1, the second combination is usually critical for limit states associated with soil failure, while the first is important in the structural design of the foundations. 
126 Consequently, in the following text, only DA1.2 and $D A 2$ will be analyzed, and, for 127 the present case, the only difference between $D A 1.2$ and $D A 2$ is the partial safety factor 128 applied to actions ( $A 1$ vs. A2).

129 It is common practice in some countries to consider a Design Approach $D A 2^{*}$ instead 130 of $D A 2$, where the effects of actions are factored instead of the actions themselves. This 131 DA2* was, however, not considered in the present paper.

\section{Finite element modeling}

The foundation analyzed in the present work represents a continuous footing with width $B$, equal to $1 \mathrm{~m}$, and subjected to a vertical load $F$. The footing was considered rigid and rough. The foundation soil was modeled with dimensions equal to $5 B \times 2 B$, in accordance with the dimensions of soil modeled in [22, 23]. Vertical and horizontal displacements on the base of the mesh and on its left and right sides were restrained. In Figure 2, the finite element mesh used is presented. This mesh consists of 16400 three node triangular elements with an element size equal to $B / 20$. The elements that represent the footing are treated as a single weightless rigid body.

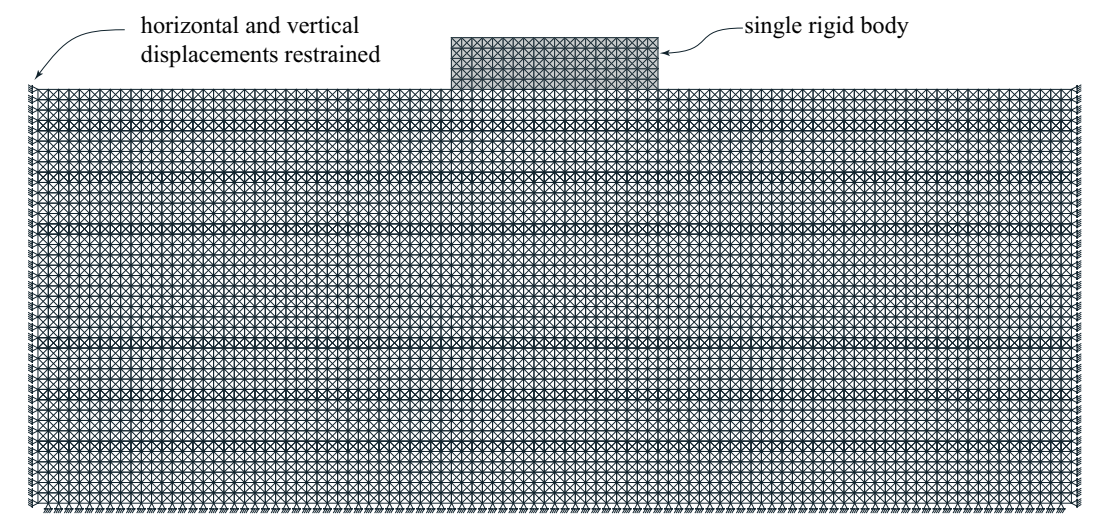

Figure 2: Mesh with a total of 16400 three node triangular elements.

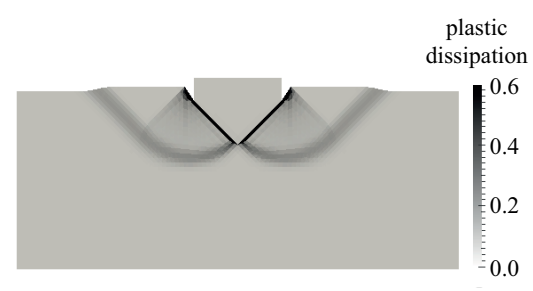

(a)

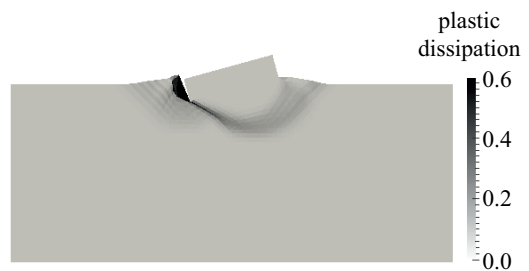

(b)

Figure 3: Collapse mechanisms using a mesh with a total of 16400 three node triangular elements for: (a) vertical centered load; (b) vertical load with an eccentricity equal to $B / 6$. 
In Figure 3 (a) the pattern of plastic dissipation considering a homogeneous soil and a centered load is presented, showing clearly the Prandtl mechanism for a foundation on homogeneous and isotropic ground. The collapse load obtained is $R_{\text {Homogeneous }}^{\text {centered }}=$ $536.1 \mathrm{kN} / \mathrm{m}$. This corresponds to a bearing capacity factor, $N_{c}$, of 5.361 , while the exact value under the conditions described is $2+\pi$, leading to the conclusion that the finite element mesh used produces an error of $4.3 \%$ compared to the exact value. For the eccentric load case (Figure 3 (b)) the mechanism for the homogeneous and isotropic ground is asymmetric, as expected, and the collapse load is $R_{\text {Homogeneous }}^{\text {eccentic }}=378.5 \mathrm{kN} / \mathrm{m}$, which corresponds to a bearing capacity factor, $N_{c}$, of 5.678 . This factor is $5.9 \%$ greater than the one obtained for centered loading. This difference can be due to the concentration of the plastic dissipation on a narrow area which would need to be more refined to obtain better results. The differences between the numerical and analytical exact results were considered admissible for the purpose of the study, as an appropriate balance between accuracy and computational time.

\section{Reliability analysis of shallow foundations}

Due to the heterogeneity of soil properties, in the present work soil strength is modelled as a random field, defined in terms of a probabilistic distribution and a correlation structure [19, e.g.]. A lognormal distribution is used along with an exponential correlation structure, described in detail in Section 5.2.

Although a range of methods have been developed over the years for evaluating structural reliability, computing the reliability of structures whose performance is evaluated using complex non-linear numerical models is still a challenge. In fact, the traditional methods for reliability analysis include simulation (i.e., Monte-Carlo simulation) and gradient-based methods (e.g, First Order Reliability Method FORM). Simulation methods are based on the generation of a large set of deterministic samples and the evaluation of the performance of each sample. The probability of failure can be computed as:

$$
p_{f}=\frac{N_{F}}{N}
$$

where $N_{F}$ is the number of samples where failure was observed and $N$ is the total number of samples. The main disadvantage of this method is the number of samples required for obtaining reliable results and the associated computational costs. In the present work, considering the computational cost of each numerical analysis, this method was excluded.

Gradient-based methods like FORM estimate the probability of failure by identifying the most likely failure point in a normalized space. A detailed description of FORM and its implementation can be found in [35]. The main disadvantage of this class of methods is the need to compute the derivatives of the performance function in order to the basic random variables. In the present work, since the performance of the foundation is computed using a complex upper bound numerical method, the accurate quantification of derivatives is impossible.

For these reasons, an hybrid approach was used in the present work. In a first step, the probability distribution of the resistance of the foundation is characterized using simulation. To reduce the required number of samples and, consequently, the computational cost, a variance reduction technique based on Latin Hypercube sampling 
was employed. Based on the results of the simulation process, a probability distribution was fit to the sampled foundation resistance. This allows the definition of the limit state function as the difference between the resistance and the effect of the applied loads. This is a simple analytical expression, and FORM can be used to compute the probability of failure.

\subsection{Simulation of random fields}

As described above, simulation can be used to generate random fields of the undrained shear strength and, finally, compute the probabilistic distribution of the collapse load. In this work, the random field was discretized using the mid-point method [30], i.e., the space was discretized in stochastic elements and a constant value of the undrained shear strength was adopted for each stochastic mesh element. This method produces a relation between the random field and a set of correlated random variables. Simulation can then be used to generate samples of the random variables and, consequently, of the associated random field.

In order to attain accurate predictions of this distribution with a minimum number of samples, Latin Hypercube sampling is used [34,36]. Although more efficient than pure Monte-Carlo simulation, this method requires a number of samples higher than the number of random variables. The simulation procedure employed followed [36]. Considering $N$ samples of a set of $K$ random variables, a matrix $\mathbf{P}_{N \times K}$ where each column corresponds to a random permutation of $1, \ldots, N$ is generated. A matrix $\mathbf{R}_{N \times K}$ of independent uniformly distributed random numbers is also generated. This method can introduce spurious correlation between the random variables and, consequently, errors in the obtained probabilistic distribution. To remove this spurious correlation and, simultaneously, to introduce the correlation structure associated with the soil properties, matrix $\mathbf{Y}$ with components $y_{i j}=\Phi^{-1}\left(\frac{p_{i j}}{N+1}\right)$ is defined, where $p_{i j}$ are the components of matrix $\mathbf{P}_{N \times K}$ and $\Phi^{-1}$ is the inverse of the standard normal cumulative distribution function. The covariance matrix of $\mathbf{Y}_{N \times K}$ is computed and Cholesky decomposed as:

$$
\overline{\mathbf{L}} \overline{\mathbf{L}}^{T}=\operatorname{Cov}(\mathbf{Y})
$$

where $\overline{\mathbf{L}}$ is lower triangular. A new matrix $\mathbf{Y}_{N \times K}^{*}$ is defined as follows:

$$
\mathbf{Y}^{*}=\mathbf{Y}\left(\overline{\mathbf{L}}^{-1}\right) \mathbf{L}^{T}
$$

where $\mathbf{L}$ corresponds to the Cholesky decomposition of the objective covariance matrix. The columns of matrix $\mathbf{P}_{N \times K}^{*}$ are formed by ranking the elements of each column of matrix $\mathbf{Y}_{N \times K}^{*}$. Finally, a sampling matrix $\mathbf{S}_{N \times K}$ can be computed as follows:

$$
\mathbf{S}=\frac{1}{N}\left(\mathbf{P}^{*}-\mathbf{R}\right)
$$

where samples are given by:

$$
x_{i j}=F_{x_{j}}^{-1}\left(s_{i j}\right)
$$

where $F_{x_{j}}^{-1}$ represent the inverse cumulative distribution associated with random variable $190 j$. 


\subsection{Probabilistic characterization of soil}

In general, the lognormal distribution is adequate to model material strengths [26]. In the case of soil properties, it is particularly useful, since variability can be very large [38], but only positive values have physical meaning [22, 6]. For these reasons, and in accordance with previous works $[22,23,28]$, the undrained shear strength was defined as a lognormal distribution.

The correlation between the soil properties in two points can be modelled considering an autocorrelation function, in terms of the vertical and horizontal distance between the two points $[52,19]$. In the present work, an ellipsoidal exponential autocorrelation function $[52,19]$ was used for the logarithm of the undrained shear strength:

$$
\rho_{l n c_{u}}\left(\Delta_{V}, \Delta_{H}\right)=\exp \left\{-\sqrt{\left(\frac{2\left|\Delta_{V}\right|}{\theta_{\ln c_{u}}^{V}}\right)^{2}+\left(\frac{2\left|\Delta_{H}\right|}{\theta_{\ln c_{u}}^{H}}\right)^{2}}\right\}
$$

where $\theta_{\ln c_{u}}^{V}$ and $\theta_{\ln c_{u}}^{H}$ are the vertical and horizontal spatial correlation lengths, respectively, and $\Delta_{V}$ and $\Delta_{H}$ are the vertical and horizontal distances between the two points. If both spatial correlation lengths are equal, the spatial correlation structure is isotropic and, otherwise, anisotropic.

The spatial correlation length, or fluctuation scale, describes the homogeneity of soil, defining a distance above which the correlation is lower than a given value [50, 52]. A high value of the correlation length implies a more homogeneous soil, with softer variations of the undrained shear strength. The correlation lengths can be replaced by a dimensionless parameter, denoted spatial correlation, defined as $\Theta_{\ln c_{u}}=\theta_{\ln c_{u}} / B$ $[22,19]$, where $B$ is the foundation width. Then, the vertical and horizontal correlation lengths can be defined in terms of the dimensionless spatial correlations, $\Theta_{l n c_{u}}^{V}=\theta_{l n c_{u}}^{V} / B$ and $\Theta_{\ln c_{u}}^{H}=\theta_{\ln c_{u}}^{H} / B$.

In this work, the mean undrained shear strength will be assumed constant and equal to $100 \mathrm{kPa}$. Considering the values proposed in literature $[33,51,5,31,24,47,12,40$, $42,38,39,14,6$, e.g.] for the mean value of the coefficient of variation and horizontal and vertical spatial correlation length of the undrained shear strength, the following values are assumed in the present work for these parameters:

$$
\begin{aligned}
& C V_{c_{u}}=\{0.125 ; 0.25 ; 0.5 ; 1\} \\
& \Theta_{l n c_{u}}^{V}=\{0.5 ; 1 ; 2 ; 4 ; 8\} \\
& \Theta_{l n c_{u}}^{H}=\left\{1.0 \Theta_{l n c_{u}}^{V} ; 10 \Theta_{\text {ln } c_{u}}^{V}\right\}
\end{aligned}
$$

The heterogeneity of the soil is modelled considering the domain divided in a quadrangular stochastic element mesh. In the generation of the samples for soil properties, four stochastic element meshes with different levels of refinement were studied as described in Table 4, with the objective of defining the minimum refinement, and, consequently, the minimum number of samples, required to ensure the correct soil spatial variability modelling. For this study, an isotropic spatial correlation structure was considered $\left(\Theta_{\ln c_{u}}^{H}=\Theta_{\ln c_{u}}^{V}\right)$, as well as a vertical centered load. In Figure 4, an example for each stochastic element mesh is presented, considering $\mathrm{CV}_{c_{u}}=0.25$ and $\Theta_{\ln c_{u}}^{V}=\Theta_{\ln c_{u}}^{H}=0.5$.

Figure 5 shows the mean and the standard deviation of the collapse load computed for different values of the coefficient of variation, $\mathrm{CV}_{c_{u}}$, and of the spatial correlation, $\Theta_{l n c_{u}}^{V}$ for the four different stochastic element meshes. Results show that a stochastic 


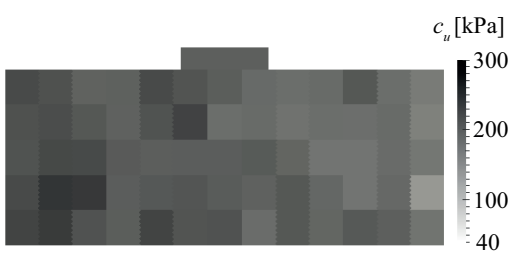

(a) $13 \times 5$

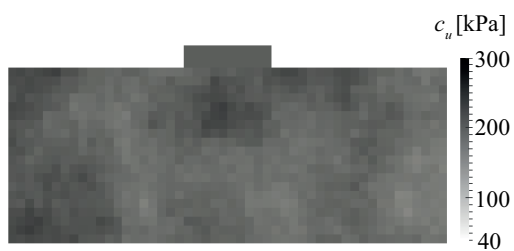

(c) $50 \times 20$

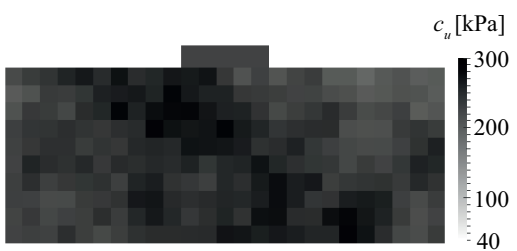

(b) $25 \times 10$

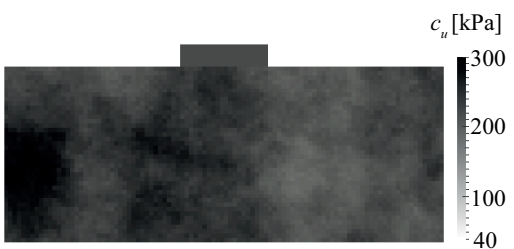

(d) $100 \times 40$

Figure 4: An example of undrained shear strength distribution for each case of stochastic element mesh studied for $\mathrm{CV}_{c_{u}}=0.25$ and $\Theta_{l n c_{u}}^{V}=\Theta_{l n c_{u}}^{H}=0.5$.

Table 4: Stochastic element meshes studied.

\begin{tabular}{cccc}
\hline $\begin{array}{c}\text { Number of } \\
\text { stochastic } \\
\text { elements }\end{array}$ & $\begin{array}{c}\text { Stochastic } \\
\text { element width } \\
d\end{array}$ & $\begin{array}{c}\text { Number of finite } \\
\text { elements inside of } \\
\text { a stochastic element }\end{array}$ & $\begin{array}{c}\text { Number } \\
\text { of samples }\end{array}$ \\
\hline $13 \times 5$ & $\sim B / 2.5$ & $\sim 256$ & 80 \\
\hline $25 \times 10$ & $B / 5$ & 64 & 300 \\
\hline $50 \times 20$ & $B / 10$ & 16 & 1200 \\
\hline $100 \times 40$ & $B / 20$ & 4 & 4800 \\
\hline
\end{tabular}

element mesh composed by elements with dimensions equal to $d=B / 10$ gives results very close to those obtained with the most refined mesh, being therefore sufficient to attain acceptable results. This mesh resulted in a minimum number of samples equal to 1000, having a total of 1200 samples been used.

\subsection{Probabilistic evaluation of bearing capacity}

Considering the simulation procedure described, the probabilistic descriptors of the collapse load for each case of soil properties were computed. In Figure 6.a and b two samples used in the simulation procedure considering a coefficient of variation $\mathrm{CV}_{c_{u}}=$ 0.25 and a vertical spatial correlation $\Theta_{l n c_{u}}^{V}=2$ are presented. Figure 6.a corresponds to an isotropic distribution of undrained shear strength $\left(\Theta_{\ln c_{u}}^{H}=\Theta_{\ln c_{u}}^{V}\right)$ and Figure 6.b to an anisotropic distribution $\left(\Theta_{l n c_{u}}^{H}=10 \Theta_{l n c_{u}}^{V}\right)$. In this case, the effect of the anisotropic correlation structure is shown by the layered distribution of properties presented. Figures 


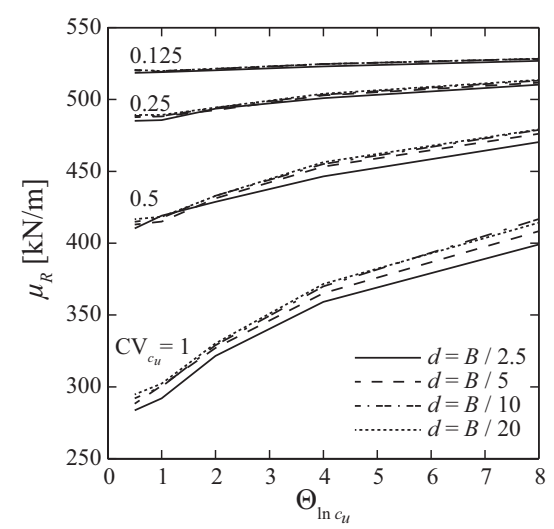

(a)

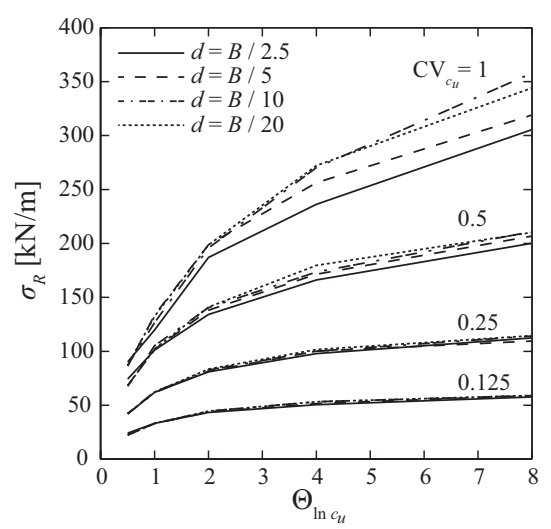

(b)

Figure 5: Evolution of mean (a) and standard deviation (b) of the bearing capacity against the value of spatial correlation for four values of the coefficient of variation and for four different stochastic element meshes, considering an isotropic spatial correlation structure.

6.c and d show the failure mechanisms for these two samples, when a centered vertical load is applied. The results obtained indicate that, even under a centered load, a significant asymmetry of the collapse mechanism can be observed, as a result of the heterogeneity of soil properties. However, probably due to the low coefficient of variation and spatial correlation considered, the differences in properties over the entire domain are relatively small and, as a result, the rotation of the foundation for the eccentric loading (see Figures 6.e and f) is compatible with that obtained considering homogeneous soil (see Figure 3).

In Figure 7, two samples with a coefficient of variation $\mathrm{CV}_{c_{u}}=1$ and a vertical spatial correlation $\Theta_{\ln c_{u}}^{V}=8$ are presented considering isotropic (left) and anisotropic (right) correlation structures. This figure, when compared to Figure 6, shows the effect of increasing the correlation length on the distribution of the undrained shear strength, denoting larger areas of higher or lower strength. In the isotropic case, an area of higher strength is clearly observable on the right side of the domain, as the left side presents low strength. As a result, the failure mode is strongly asymmetric, with energy dissipation concentrated on the left side. In all cases, mechanisms show much greater complexity, as the failure surface crosses areas of lower undrained shear strength.

The mean normalized collapse load and the standard deviation of the collapse load computed for different values of the coefficient of variation, $\mathrm{CV}_{c_{u}}$, and the vertical spatial correlation, $\Theta_{\ln c_{u}}^{V}$, are presented in Figures 8 and 9, for isotropic and anisotropic correlation structures, respectively. The normalized collapse load is determined by dividing the obtained load by that determined in Section 4 associated with a homogeneous soil characterized by the mean undrained strength subjected to the same loading.

As previously mentioned, the bearing capacity of shallow foundations subjected to a centered vertical load and on soils responding in undrained conditions was studied in [22, $23,41,13,28,9,27]$. These works showed that the soil spatial variability can significantly change the collapse mechanism and the bearing capacity. They also concluded that the normalized collapse load is lower than 1 . Consistent observations are presented in 


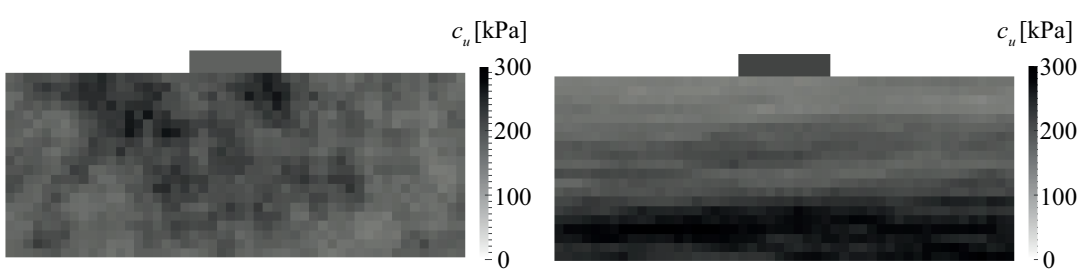

(a)

(b)

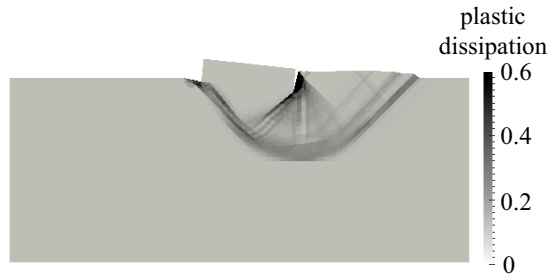

(c)

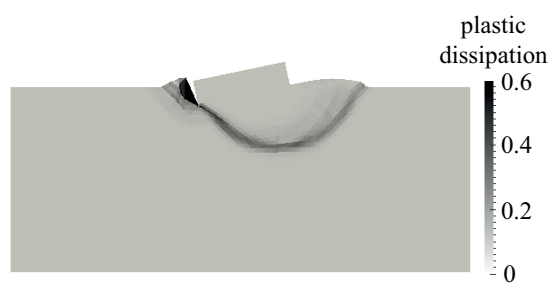

(e)

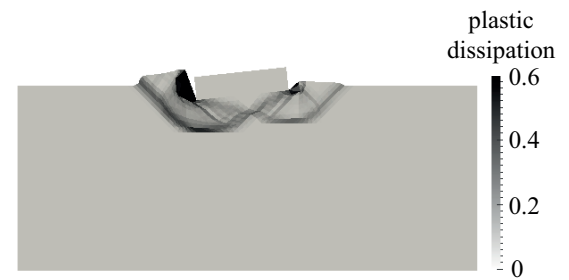

(d)

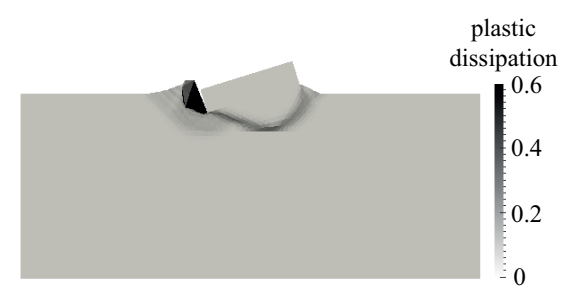

(f)

Figure 6: Examples of collapse mechanisms for both centered (c and d) and eccentric load (e and f) corresponding to an example of an undrained shear strength distribution (a and b) considering an isotropic (left) and an anisotropic spatial correlation structure (right) and for $\mathrm{CV}_{c_{u}}=0.25$ and $\Theta_{l n c_{u}}^{V}=$ 2 .

this work for centered and eccentric loads, as well as isotropic and anisotropic spatial correlation structures.

In fact, the results obtained in this section are justified by the observation of $[22,23]$ stating that the presence of weak zones in the region of interest governs the failure mechanism and, therefore, the bearing capacity of shallow foundations. The results presented show that increased uncertainty (higher $\mathrm{CV}_{c_{u}}$ ) significantly reduces the mean resistance, while increasing the collapse load standard deviation. Moreover, heterogeneity (associated with low correlation lengths) leads to a reduction of the mean resistance. In fact, more heterogeneous soils are more likely to present areas of lower strength in the region of interest, and will be associated with failure mechanisms through these elements. The standard deviation of the bearing capacity increases with the correlation length. For highly correlated soil properties, large areas of lower strength exist. If, on one hand, these are located in the region of interest, a very low bearing capacity is obtained. On the other hand, if an area of higher strength is located in the region of interest, a much 


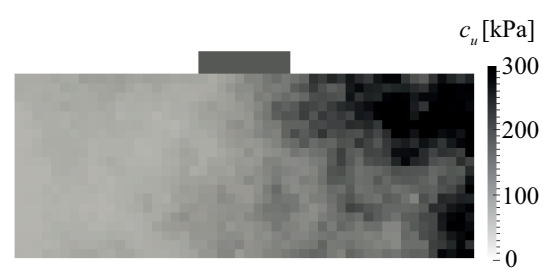

(a)

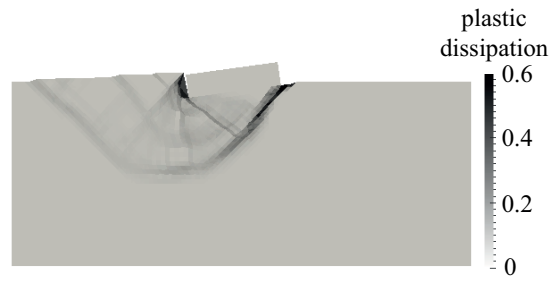

(c)

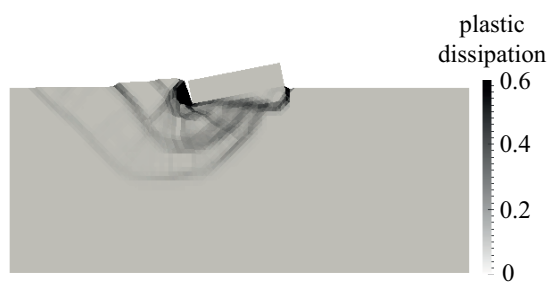

(e)

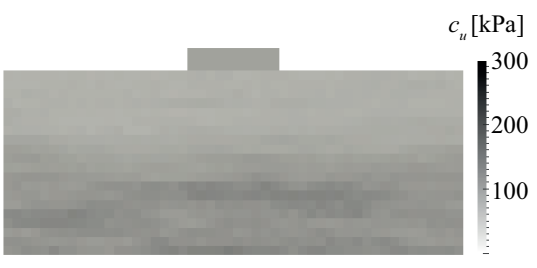

(b)

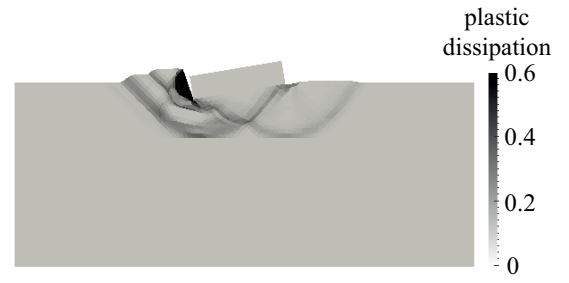

(d)

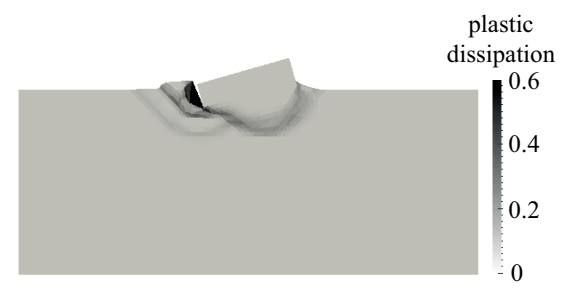

(f)

Figure 7: Collapse mechanisms for both centered (c and d) and eccentric load (e and f) corresponding to an example of an undrained shear strength distribution (a and b) considering an isotropic (left) and an anisotropic spatial correlation structure (right) and for $\mathrm{CV}_{c_{u}}=1$ and $\Theta_{l n c_{u}}^{V}=8$.

higher bearing capacity results. Consequently, a larger dispersion in the distribution of the bearing capacity is obtained in this case. The difference is larger for higher coefficients of variation, as these result in greater differences between areas of lower and higher strength. The results for the anisotropic cases (Figure 9) show that an increase in the horizontal spatial correlation leads to an increase of both mean and standard deviation of the bearing capacity, which is consistent with results presented in Figure 8 .

Although the bearing capacity under eccentric loads is lower than that obtained for centered loads, the results in Figures 8 and 9 show that the consideration of soil uncertainty has a lower impact for eccentric load scenarios, proved by the higher mean normalized bearing capacity obtained for eccentric loads. This is particularly relevant for lower coefficients of variation, for which a mean normalized capacity very close to 1.00 is obtained. In terms of standard deviation, the results presented show little impact of the eccentricity of loads for lower values of $C V_{c_{u}}$; it increases for higher coefficients of variation and higher spatial correlation.

The results presented show that within the range of values analyzed: (i) increasing 


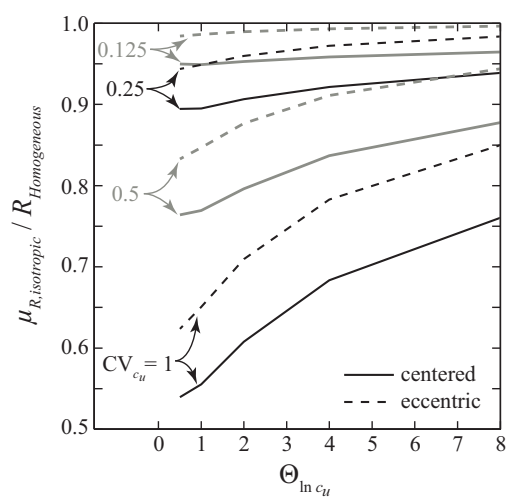

(a)

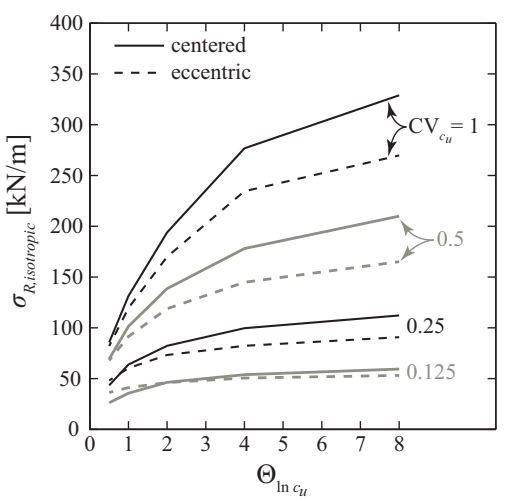

(b)

Figure 8: Evolution of normalized mean (a) and standard deviation (b) of bearing capacity against the value of spatial correlation for four coefficient of variation values and for both centered and eccentric load (as defined in Figure 1), considering an isotropic spatial correlation structure.

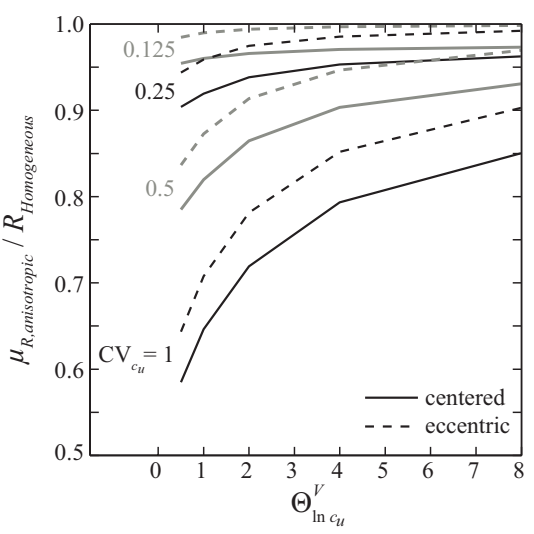

(a)

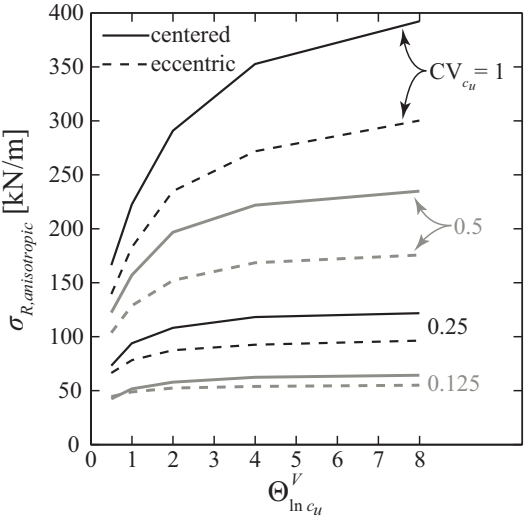

(b)

Figure 9: Evolution of normalized mean (a) and standard deviation (b) of bearing capacity against the value of spatial correlation for four coefficient of variation values and for both centered and eccentric load, considering an anisotropic spatial correlation structure with $\Theta_{\ln c_{u}}^{H}=10 \Theta_{\ln c_{u}}^{V}$.

the coefficient of variation results in lower mean values of bearing capacity; (ii) increasing spatial correlation leads to an increase of mean values of bearing capacity; (iii) higher values of the coefficient of variation and spatial correlation result in higher standard deviation of the bearing capacity. The results also show that these conclusions are valid for both centered and eccentric loads. The results obtained for the case of centered loading and isotropic spatial correlation structure are coherent with previous works on the topic $[22,23,28]$, showing the consistency of the methodology applied in this work (combination of Sublim3d with Latin Hypercube sampling) to calculate the response of 
shallow foundations.

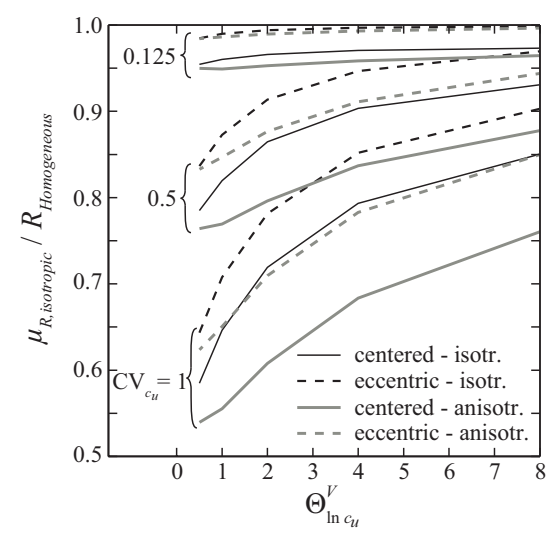

(a)

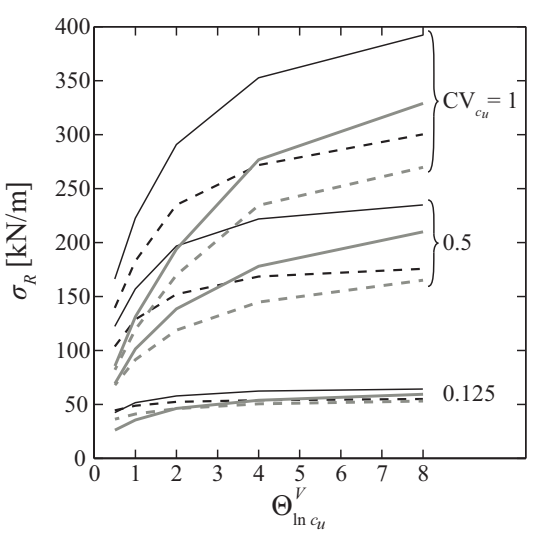

(b)

Figure 10: Comparison between the normalized mean (a) and the standard deviation (b) of bearing capacity against the value of spatial correlation for four coefficient of variation values and for both centered and eccentric load, considering an isotropic and anisotropic spatial correlation structure.

The comparison of results obtained for both correlation structures shows that the anisotropic spatial correlation structure results in higher values of both mean and standard deviation of the bearing capacity (see Figure 10). In fact, the anisotropic structure is associated with a higher horizontal spatial correlation and, as previously observed, higher spatial correlation result in an increase of bearing capacity mean value and standard deviation.

\section{Reliability assessment of shallow foundations}

The probabilistic analysis presented showed that the load eccentricity and coefficient of variation, correlation length and spatial correlation structure of the undrained shear strength have different, and in some cases conflicting effects, on the mean and standard deviation of the bearing capacity. A simple relation between the safety of foundations and these probabilistic descriptors is not possible. Therefore, a parametric reliability analysis was performed to analyse the dependence of the reliability index on the probabilistic descriptors of soils.

In this section, the structural safety of shallow foundations is analyzed with a reliability framework for foundations designed following Eurocode 7 [11]. The limit state function, $Z$, was defined as:

$$
Z(R, G, Q)=R-G-Q
$$

where $R$ is the bearing capacity, $G$ is the permanent load and $Q$ is the live load. The bearing capacity was modelled by fitting an appropriate probability distribution for each set of results obtained in the previous section. The permanent and live load were modeled using a normal and a Gumbel distribution, respectively [7, 26, 49, 25, e.g.]. This analysis 
323 presents a practical interest as it evaluates the consistency of the partial safety factors method, currently prescribed in Eurocode 7[11].

To model the bearing capacity, normal, lognormal, exponential, Gamma and Weibull distributions were fitted to the results described above. The probabilistic models were compared using the maximum log-likelihood function. An example of the results obtained is presented in Figure 11, where the different distribution models are fitted to the bearing capacity computed considering an isotropic spatial correlation structure with $\mathrm{CV}_{c_{u}}=0.5$ and $\Theta_{\ln c_{u}}=4$ and a centered load. The results obtained for all the scenarios analyzed showed that the lognormal distribution resulted in adequate fit in all cases and, for this reason, was used to model the bearing capacity.

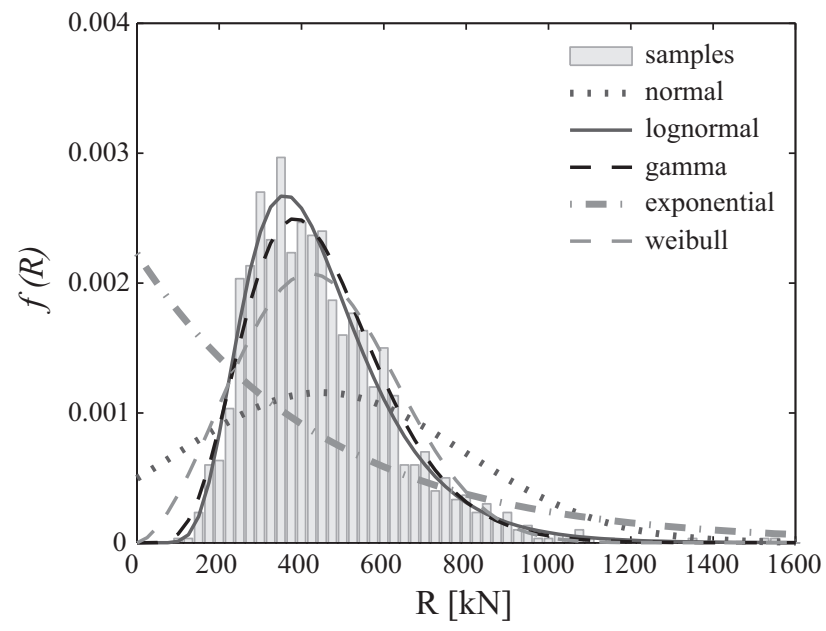

Figure 11: Foundation resistance: histogram and fitted probability distributions for the case study corresponding to an isotropic spatial correlation structure with $\mathrm{CV}_{c_{u}}=0.5$ and $\Theta_{\ln c_{u}}=4$ and for centered load.

The reliability analysis of the foundation was performed for each distribution of the undrained shear strength and each Eurocode Design Approach, considering different ratios between dead and live loads $\left(Q_{k} /\left(Q_{k}+G_{k}\right)\right)$. Each distribution of the undrained shear strength results in a different nominal value of strength, $c_{u, k}$ computed herein as the $5 \%$ percentile of the associated distribution. Based on this and on the partial safety factors associated with each Design Approach, the design foundation resistance $R_{d}$ can be computed as:

$$
R_{d}=(2+\pi) \cdot B^{\prime} \cdot \frac{c_{u, k}}{\gamma_{c_{u}}} \cdot \frac{1}{\gamma_{R ; v}}
$$

Based on this and the the ratios between dead and live loads, the maximum nominal oads which can safely be applied, according to Eurocode 7 [11], are:

$$
A_{d}=\gamma_{G} \cdot G_{k}+\gamma_{Q} \cdot Q_{k} \leq R_{d}
$$

From these, the probability distribution of the dead load and the live load to be used in the reliability analysis can be computed assuming that the permanent load is 
characterized by a normal distribution with a coefficient of variation equal to $10 \%$ while the live load can be modeled appropriately by a Gumbel distribution with a coefficient of variation equal to $35 \%[7,26,49,25$, e.g.]. If all variables were independent and normally distributed, the reliability index could be computed as:

$$
\beta=\Phi^{-1}\left(p_{f}\right)=\frac{\mu_{R}-\mu_{G}-\mu_{Q}}{\sqrt{\sigma_{R}^{2}+\sigma_{G}^{2}+\sigma_{Q}^{2}}}
$$

where $\mu_{R}, \mu_{G}$ and $\mu_{Q}$ are the mean of the normal distribution which characterize the resistance, permanent load and live load, respectively, and $\sigma_{R}, \sigma_{G}$ and $\sigma_{Q}$ are the correspondent standard deviations. In the present case, as the variables are non-Gaussian, this expression results only in an estimate of the reliability index. Consequently, the reliability index was computed using iForm [48], implemented in the open-source program FERUM (Finite Element Reliability Using Matlab) [8, 29].

Figure 12 shows the reliability index obtained considering both Design Approaches and an isotropic spatial correlation structure. The obtained reliability indices vary significantly, as wide ranges of coefficients of variation $\left(C V_{c_{u}}\right)$ and spatial correlation $\left(\Theta_{\ln c_{u}}\right)$ are considered.

The results show, as expected, that increasing the coefficient of variation $\left(C V_{c_{u}}\right)$ or the spatial correlation $\left(\Theta_{\ln c_{u}}\right)$ leads to a reduction in the reliability index. In fact, as shown in Figure 10, increasing either of these parameters leads to an increase in the standard deviation of the bearing capacity, which, considering equation 14, results in a reduction of the reliability index. These results also show that the ratio between live and total load influences the reliability index. However, except for very low spatial correlation $\left(\Theta_{l n c_{u}}=0.5\right)$ or very low coefficients of variation of the undrained shear strength $\left(C V_{c_{u}}=0.125\right)$, the reliability index is relatively uniform, showing that the partial safety factors for loads defined in Eurocode 7 [11] are adequate.

The decrease of the reliability index with the increasing of the spatial correlation is more evident for the isotropic than for the anisotropic correlation structure, which results from the greater influence of this parameter in the standard deviation value of bearing capacity for the first type of correlation structure (see Figure 10). It can also be seen that the trend previously mentioned (i.e. higher values of reliability index for lower values of spatial correlation) is more evident for higher values of the coefficient of variation, which also has the same explanation. For both structures and both loads, the influence of the spatial correlation is higher for greater values of the coefficient of variation.

Comparing the two Design Approaches considered, the only difference, for the structure under analysis, lies on the lower load partial safety factors used for DA1.2. From equation 13, this results in higher mean loads and, consequently, higher standard deviations of loads for DA1.2. This increase in mean and standard deviation of design foundation resistance leads to a lower reliability index (see equation 14). Since in equation 14 the square root of the sum of the squared standard deviations is considered, the effect of the increase in standard deviation of loads is reduced when the uncertainty in load bearing capacity $\left(\sigma_{R}\right)$ increases. As a result, as shown in Figure 12, the reliability indices obtained using $D A 1.2$ (left) are always lower than those resulting from $D A 2$ (right), but this difference is reduced for higher coefficients of variation and spatial correlations.

The results obtained considering an anisotropic correlation structure (Figure 13) show 


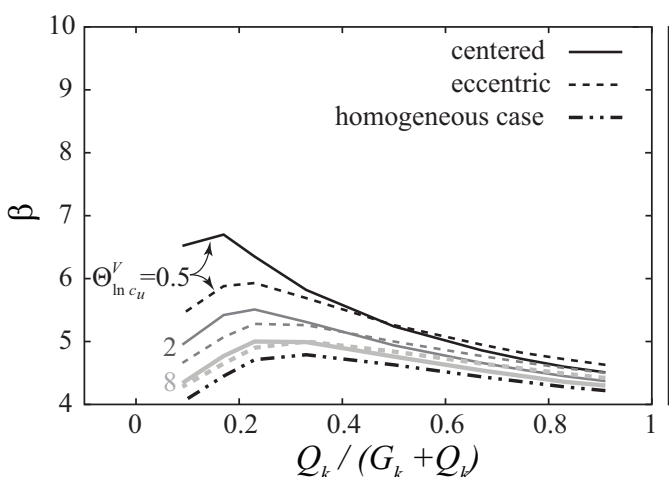

(a)

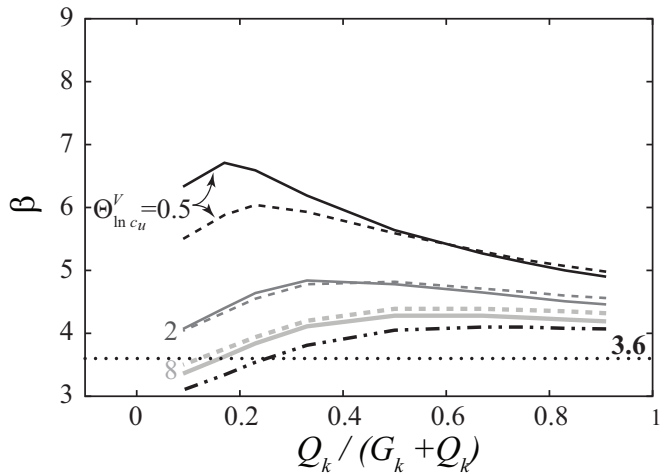

(c)

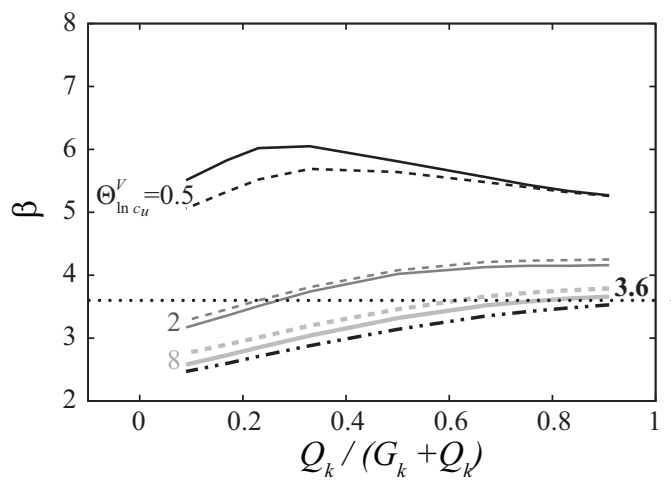

(e)

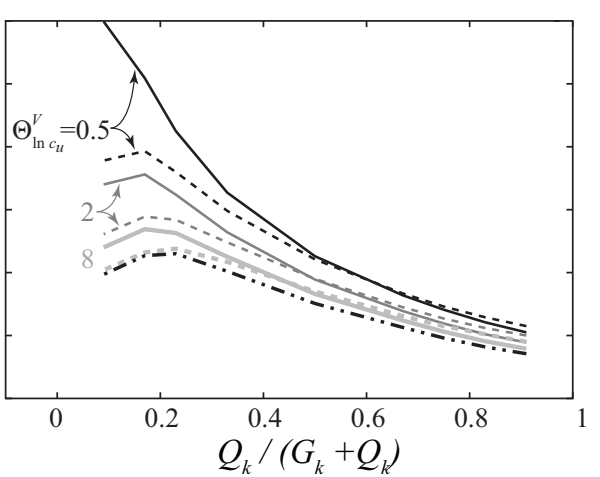

(b)

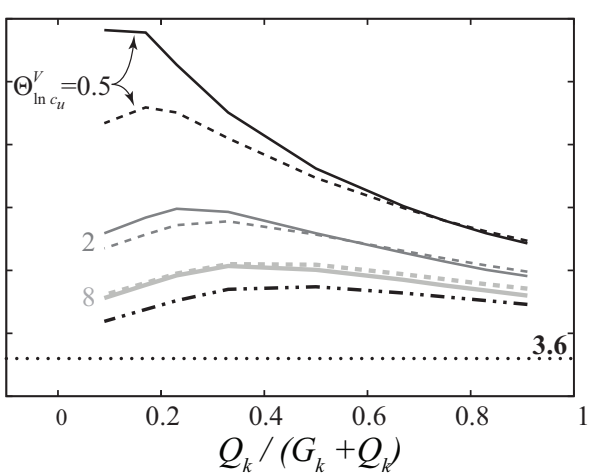

(d)

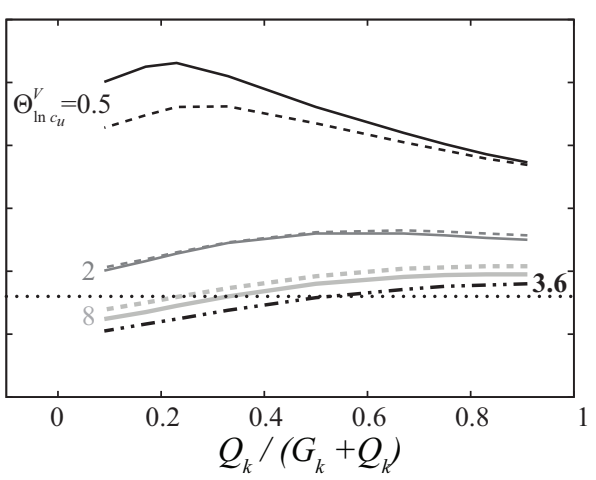

(f)

Figure 12: Evolution of reliability index against loads ratio for an isotropic spatial correlation structure with $C V_{c_{u}}$ equal to 0.125 (a and b), 0.25 (c and d) and 0.5 (e and f), considering the DA1.2 (left) and the $D A 2$ (right). 


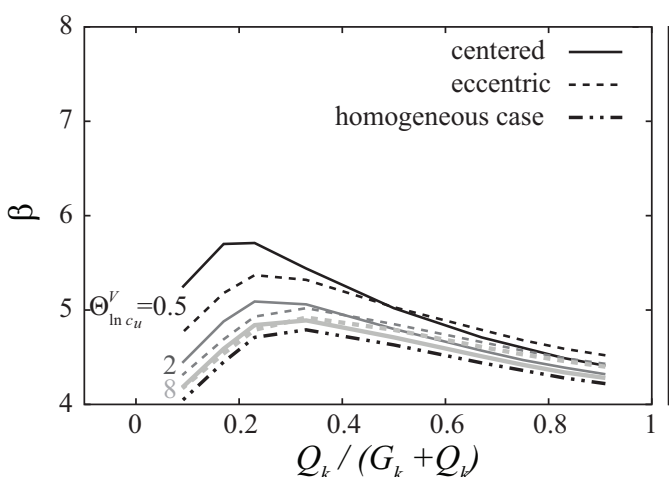

(a)

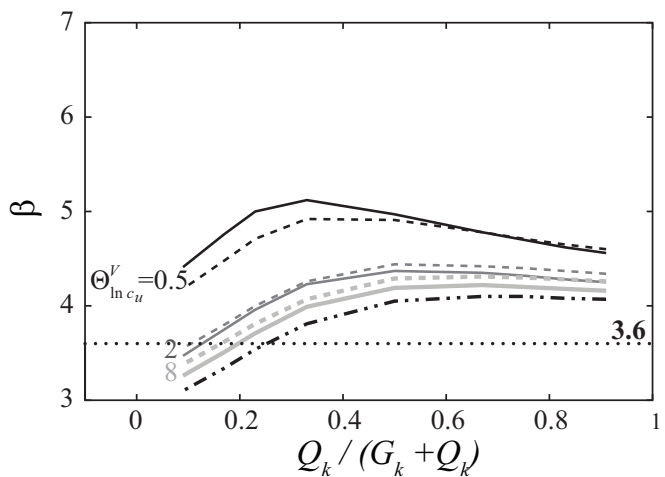

(c)

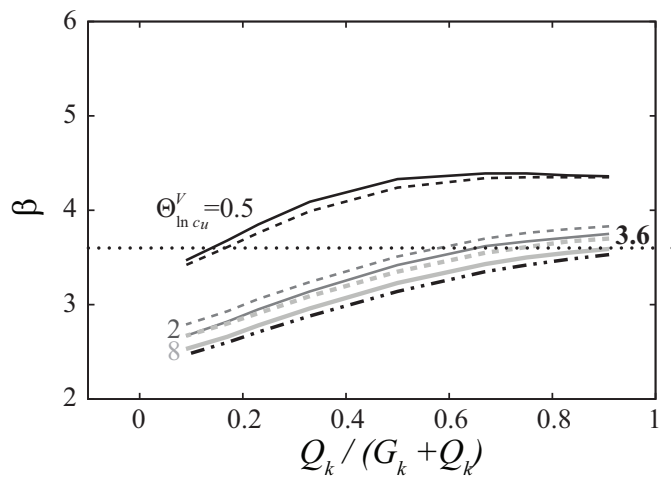

(e)

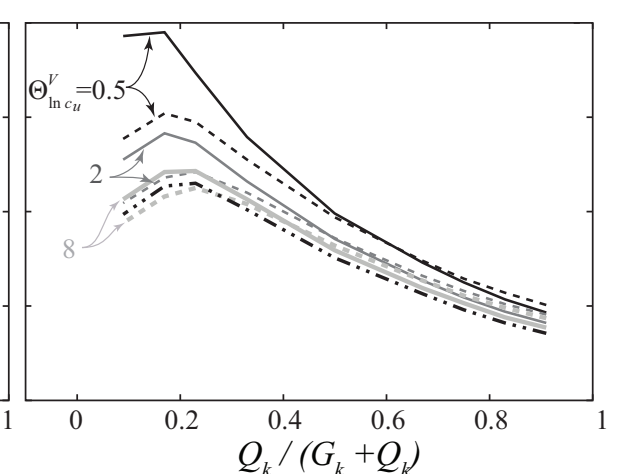

(b)

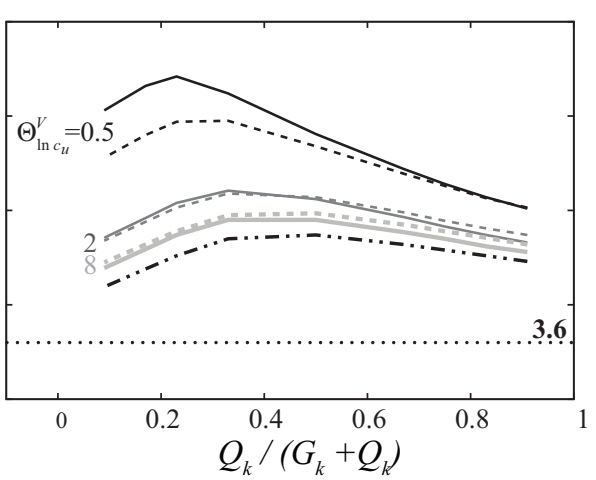

(d)

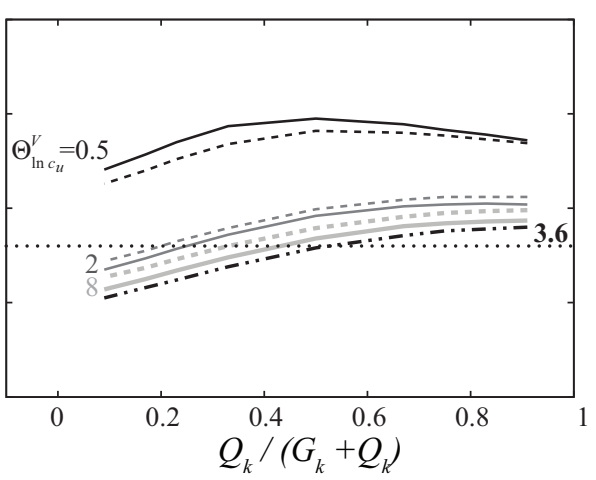

(f)

Figure 13: Evolution of reliability index against loads ratio for an anisotropic spatial correlation structure with $C V_{c_{u}}$ equal to 0.125 (a and b), 0.25 (c and d) and 0.5 (e and f), considering the DA1.2 (left) and the $D A 2$ (right).

384 The comparison of the results obtained under a centered and an eccentric load show 
very small differences, indicating that the approach used in Eurocode 7 [11] for assessing the safety of eccentric loaded shallow foundations is consistent.

In Figures 12 and 13, the reliability index obtained assuming a homogeneous soil $\left(\theta_{\ln c_{u}} \rightarrow \infty\right)$ and an analytical limit state function are also presented. In all examples analyzed, assuming a homogeneous soil leads to lower safety levels, as a result of the increase in bearing capacity standard deviation. Therefore, analytical models assuming homogeneous soil can be regarded as a simple but conservative approach to the safety analysis of shallow foundation.

Comparing the reliability indices computed with the threshold defined in Eurocode 0 [10] $\left(\beta_{\text {target }}=3.6\right)$, it can be seen that $D A 2$ leads to acceptable safety levels, except if lower values of live load to total load ratios, high coefficient of variation and spatial correlation are considered for the undrained shear strength. On the other hand, the use of Design Approach DA1.2 leads to undesirable safety levels for a wide range of probabilistic soil properties, presenting the same trends as seen for the Design Approach $D A 2$.

\section{Conclusions}

In the present work a reliability assessment of shallow foundations subjected to vertical loads, responding in undrained conditions, considering explicitly the soil spatial variability was carried out. Firstly, random fields representing the soil, considering different coefficients of variation, both vertical and horizontal spatial correlation lengths and soil spatial correlation structure were generated using Latin Hypercube sampling. Regarding to the shallow foundations bearing capacity response, it is important to highlight that:

- the mean value of the bearing capacity increases with decreasing coefficients of variation and increasing spatial correlation lengths. The standard deviation value of the bearing capacity increases with increasing coefficients of variation and spatial correlation lengths. These trends were observed for both centered and eccentric loadings, as well as isotropic and anisotropic distributions of the spatial variability. These observations are consistent with previous works focusing on the same topic $[22,23,41,13,28,9,27]$, which confirms that the combination of the Latin Hypercube sampling $[34,36]$ with Sublim3d [45] is suitable to investigate the influence of soil heterogeneity in the bearing capacity of foundations [46];

- the ratio between the mean value of the bearing capacity and the bearing capacity value determined considering a homogeneous soil is similar for centered and eccentric loads. Regarding to the standard deviation, the values obtained tend to be smaller for the eccentric load;

- the results obtained for an anisotropic spatial correlation structure are consistent with those expected when there is an increase in the spatial correlation length, i.e., comparing the results obtained for both spatial correlation structures, greater values of mean and standard deviation values of the bearing capacity are verified for the anisotropic structure (for the same vertical scale of fluctuation). 
With the bearing capacity response reliability index following Eurocode 7 [11] approaches were calculated based on iForm [48] implemented on the open-source program FERUM [8]. With respect to the results obtained for the reliability index, the main conclusions are:

- DA2 leads always to larger values of reliability index as consequence of its greater values for partial safety factors;

- DA1.2 leads to undesirable safety levels for a wide range of probabilistic soil properties, presenting, nevertheless, the same trends as seen for the Design Approach $D A 2$;

- the difference between the two approaches will increase if the base of the foundation is below the surface, as is usually the case in practice;

- the results have shown very small differences between centered and eccentric load;

- the approach used in Eurocode 7 [11] for assessing the safety of eccentric loaded shallow foundations is consistent;

- for the cases analysed in the present work, reliability analysis using analytical models assuming homogeneous soil can be regarded as a simple but conservative approach to the safety analysis of shallow foundations;

\section{Acknowledgments}

The authors acknowledge the support of the Geotechnical Group of the Department of Civil Engineering of the Universidade Nova de Lisboa and UNIC - Centro de Investigação em Estruturas e Construção. The second author acknowledges the support of Fundação para a Ciência e Tecnologia through grant PTDC/ECM/115932/2009.

\section{References}

[1] Ahmed, A., Soubra, A.H.: Probabilitstic analysis of strip footings resting on a spatially random soil using subset simulation approach. Georisk 6, 188-201 (2012)

[2] Ahmed, A., Soubra, A.H.: Probabilitstic analysis at serviceability limit state of two neigboring strip footings resting on a spatially random soil. Structural Safety 49, 2-9 (2014)

[3] Al-Bittar, T., Soubra, A.H.: Probabilistic analysis of strip footings resting on spatially varying soils and subjected to vertical or inclined loads. Journal of Geotechnical and Geoenvironmental Engineering 140(4) (2014)

[4] Antão, A.N., Vicente da Silva, M., Guerra, N.M.C., Delgado, R.: An upper bound-based solution for the shape factors of bearing capacity of footings under drained conditions using a parallelized mixed f.e. formulation with quadratic velocity fields. Computers and Geotechnics 41, 23-35 (2012)

[5] Asoaka, A., Grivas, D.: Spatial variability of the undrained strength of clays. Journal of Engineering Mechanics 108(5), 743-756 (1982). ASCE

[6] Baecher, G.B., Christian, J.T.: Reliability and Statistics in Geotechnical Engineering. John Wiley and Sons (2003)

[7] Bartlett, F.M., Hong, H.P., Zhou, W.: Load factor calibration for the proposed 2005 edition of the national building code of canada: Statistics of loads and load effects. Canadian Journal of Civil Engineering 30(2), 429-439 (2003)

[8] Bourinet, J.M.: Ferum 4.1 user's guide (2010)

[9] Cassidy, M.J., Uzielli, M., Tian, Y.: Probabilistic combined loading failure envelopes of a strip footing on spatially variable soil. Computers and Geotechnics 49, 191-205 (2013) 
[10] CEN: EN 1990:2009, Eurocode 0 - Basis of structural design. European Committee for Standardization (2009)

[11] CEN: EN 1997-1:2010, Eurocode 7 - Geotechnical design. European Committee for Standardization (2010)

[12] Chiasson, P., Lafleur, J., Soulié, M., Law, K.T.: Characterizing spatial variability of a clay by geostatistics. Canadian Geotechnical Journal 32, 1-10 (1995)

13] Cho, S.E., Park, H.C.: Effect of spatial variability of cross-correlated soil properties on bearing capacity of strip footing. International Journal for Numerical and Analytical Methods in Geomechanics 34, 1-26 (2010)

[14] Duncan, J.M.: Factors of safety and reliability in geotechnical engineering. Journal of Geotechnical and Geoenvironmental Engineering 126, 307-316 (2000)

[15] Fenton, G.A.: Simulation and analysis of random fields. Ph.D. thesis, Department of Civil Engineering and Operations Research, Princeton University (1990)

[16] Fenton, G.A.: Error evaluation of three random-field generators. Journal of engineering mechanics 120(12), 2478-2497 (1994)

[17] Fenton, G.A., Griffiths, D.V.: Probabilistic foundation settlement on a spatially random soil. Journal of Geotechnical and Geoenvironmental Engineering 128(5), 381-390 (2002). ASCE

[18] Fenton, G.A., Griffiths, D.V.: Three-dimensional probabilistic foundation settlement. Journal of Geotechnical and Geoenvironmental Engineering 131(2), 232-239 (2005). ASCE

[19] Fenton, G.A., Griffiths, D.V.: Risk Assessment in Geotechnical Engineering. John Wiley and Sons, New York (2008)

[20] Fenton, G.A., Vanmarcke, E.: Simulation of random fields via local average subdivision. Journal of Engineering Mechanics 116(8), 1733-1749 (1990). ASCE

[21] Florian, A.: An efficient sampling scheme: Updated latin hypercube sampling. Probabilistic Engineering Mechanics 7, 123-130 (1992)

[22] Griffiths, D.V., Fenton, G.A.: Bearing capacity of spatially random soil: the undrained clay prandtl problem revisited. Géotechnique 51(4), 351-359 (2001)

[23] Griffiths, D.V., Fenton, G.A., Manoharan, N.: Bearing capacity of rough rigid strip footing on cohesive soil: Probabilistic study. ASCE Journal Geotechnical and Geoenvironmental Engineering 128(9), 743-755 (2002)

[24] Harr, M.E.: Reliability based design in civil engineering. McGraw Hill, London, New York (1987)

[25] Holicky, V., Markova, J., Gulvanessian, H.: Code calibration allowing for reliability differentiation and production quality. In: Application of Statistics and Probability in Civil Engineering: Proceedings of the 10th International Conference. Kanda, Takada and Furuat (2007)

[26] JCSS: JCSS Probabilistic Model Code. Part 2: Load Models. Joint Commitee on Structural Safety (2001)

[27] Jha, S.: Reliability-based analysis of bearing capacity of strip footings considering anisotropic correlation of spatially varying undrained shear strength. International Journal of Geomechanics (2016)

[28] Kasama, K., Whittle, A.J.: Bearing capacity of spatially random cohesive soil using numerical limit analyses. Journal of Geotechnical and Geoenvironmental Engineering 137(11), 989-996 (2011)

[29] Kiureghian, A.D., Haukaas, T., Fujimura, K.: Structural reliability software at the university of california, berkeley. Structural Safety 28(1), 44-67 (2006)

[30] Kiureghian, A.D., Ke, J.B.: The stochastic finite element method in structural reliability. Probabilistic Engineering Mechanics 3(2), 83-91 (1988)

[31] Lee, I., White, W., Ingles, O.: Geotechnical Engineering. Pitman, London (1983)

[32] Low, B.K., Phoon, K.K.: Reliability-based design and its complementary role to eurocode 7 design approach. Computers and Geotechnics 65, 30-44 (2015)

[33] Lumb, P.: The variability of natural soils. Canadian Geotechnical Journal 3, 74-97 (1966)

[34] McKay, M.D., Beckman, R.J., Conover, W.J.: A comparison of three methods for selecting values of input variables in the analysis of output from a computer code. Technometrics 21(2), 239-245 (1979)

[35] Melchers, R.E., Beck, A.T.: Structural reliability analysis and prediction. John Wiley \& Sons (2018)

[36] Olsson, A., Sandberg, G., Dahlblom, O.: On latin hypercube sampling for structural reliability analysis. Structural Safety 25(1), 47-68 (2003)

[37] Paice, G.M., Griffiths, D.V., Fenton, G.A.: Finite element modeling of settlements on spatially random soil. Journal of Geotechnical Engineering 122(9), 777-779 (1996)

[38] Phoon, K.K., Kulhawy, F.H.: Characterization of geotechnical variability. Canadian Geotechnical 


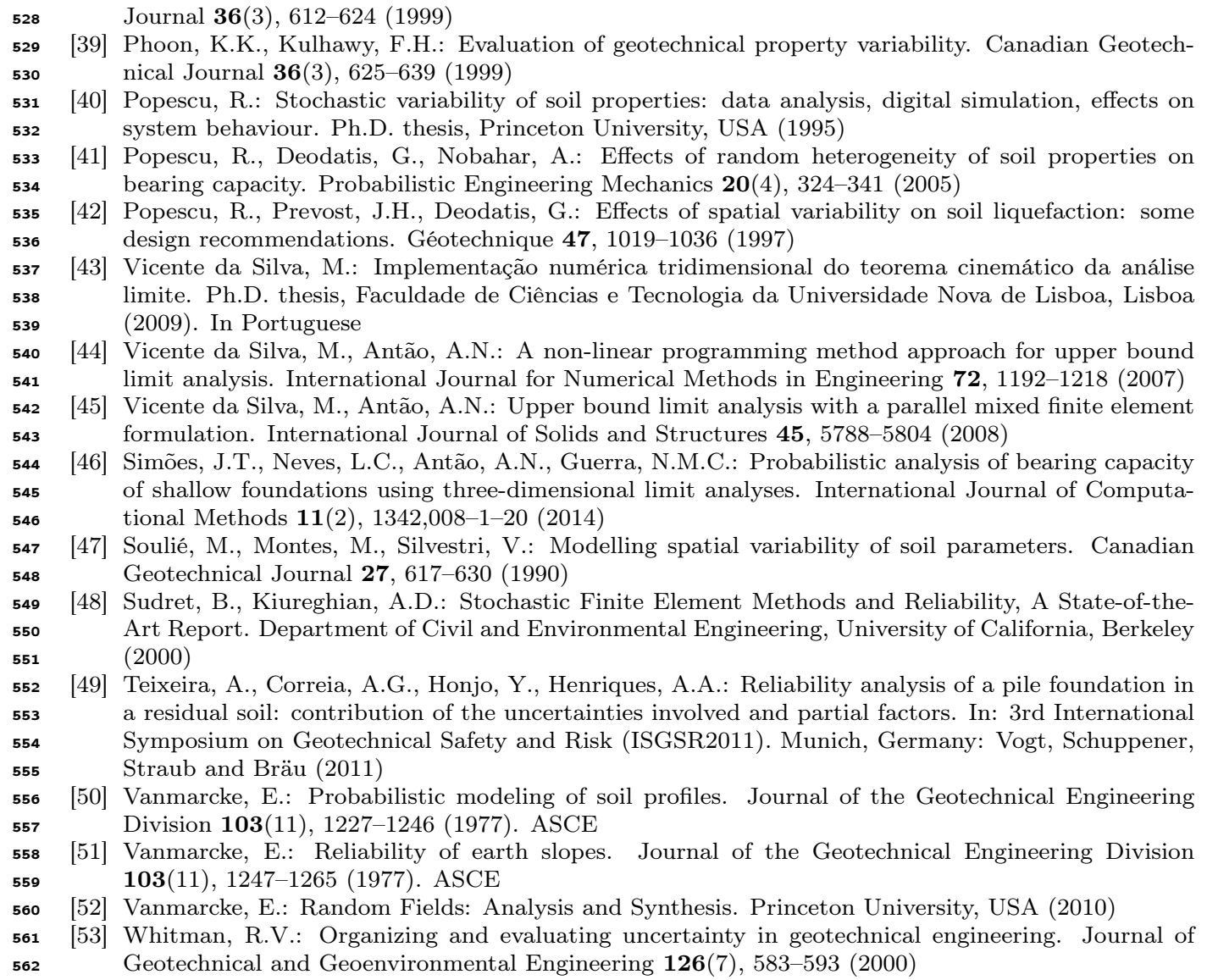

